\title{
Operator-guided Navigator Gating for Real-Time Interactive Coronary Cardiovascular Magnetic Resonance
}

\author{
Keigo Kawaji ${ }^{*}$, Mita Patel', Jouke Smink², Hui Wang ${ }^{3}$, Roberto Lang ${ }^{1}$, Amit R Patel ${ }^{1}$ \\ From 19th Annual SCMR Scientific Sessions \\ Los Angeles, CA, USA. 27-30 January 2016
}

\section{Background}

Real-time interactive (RTI) MRI parameter manipulation during the scan [1] may potentially address challenges imposed by respiratory motion during a free-breathing cardiovascular magnetic resonance (CMR) acquisition. In this study, we propose an operator-guided processing that allows manipulation of navigator gating parameters in real-time. This approach was evaluated in healthy volunteers, where coronary CMR (CCMR) with and without RTI manipulation was examined to assess acquisition failure rates, scan time reduction, and vessel sharpness.

\section{Methods}

The proposed RTI framework employs a custom communication protocol between the scanner host and the waveform generation hardware that allows non-time-critical operator tasks (ie. made changes are reflected in the next collected heart beat without overtasking the scan runtime). The custom front-end (Figure 1a) shows the operator-interactive navigator control that allows manipulation of the diaphragm navigator gating window in real-time (Figure 1b).

This RTI approach was incorporated into a CCMR sequence with view/profile order compatible with

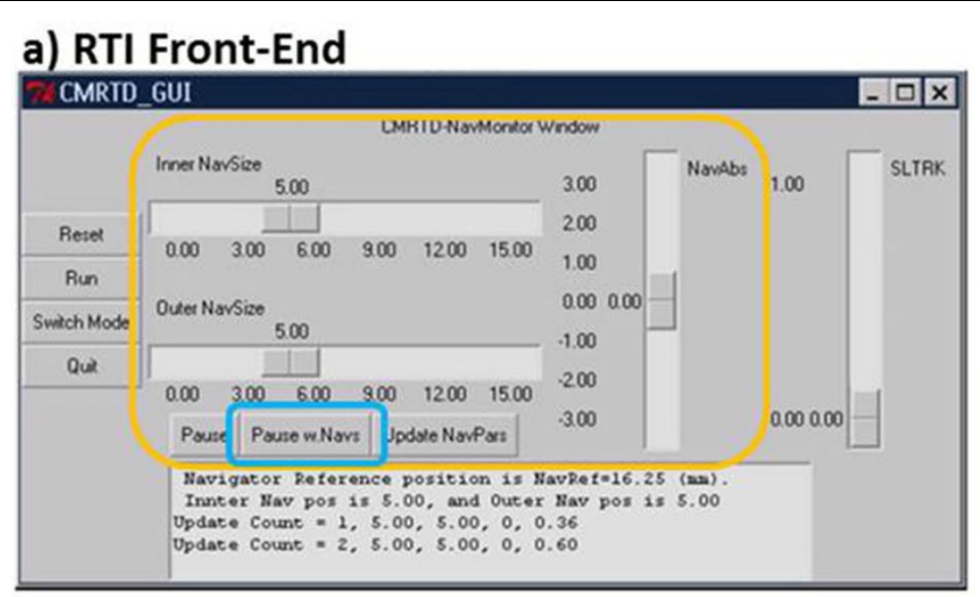

\section{b) Navigator Control Examples}

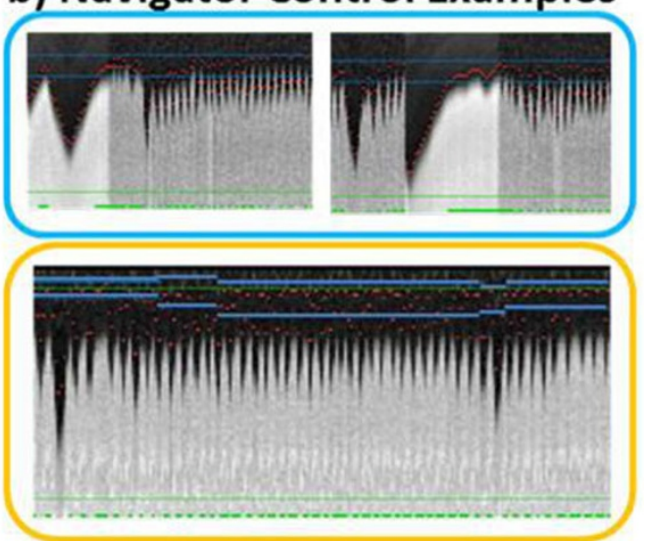

Figure 1 Real-time and Interactive control of navigator parameters. a) Graphical User Interface. b) Operator control examples - blue: of manual pause and repeated navigator during breath-hold instruction (used in $n=1$ subject in this study); orange: interactive manipulation of navigator gating window position parameters (used in all 13 subjects). This example shows the operator manually adjusting the gating window to the subject's respiratory drift position.

\footnotetext{
Medicine, Section of Cardiology, The University of Chicago, Chicago, IL, USA
} Full list of author information is available at the end of the article 

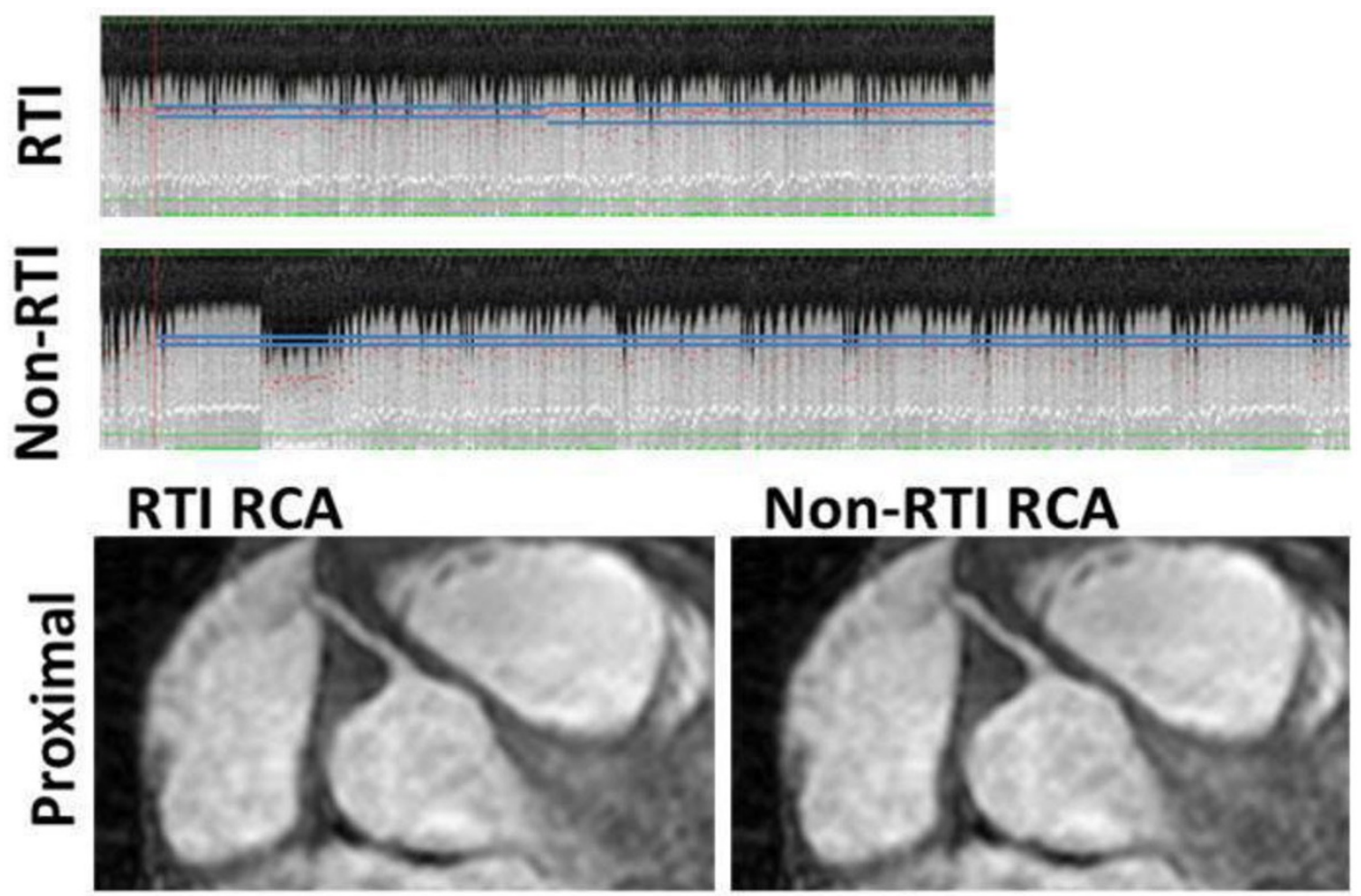

\section{Non-RTI RCA}
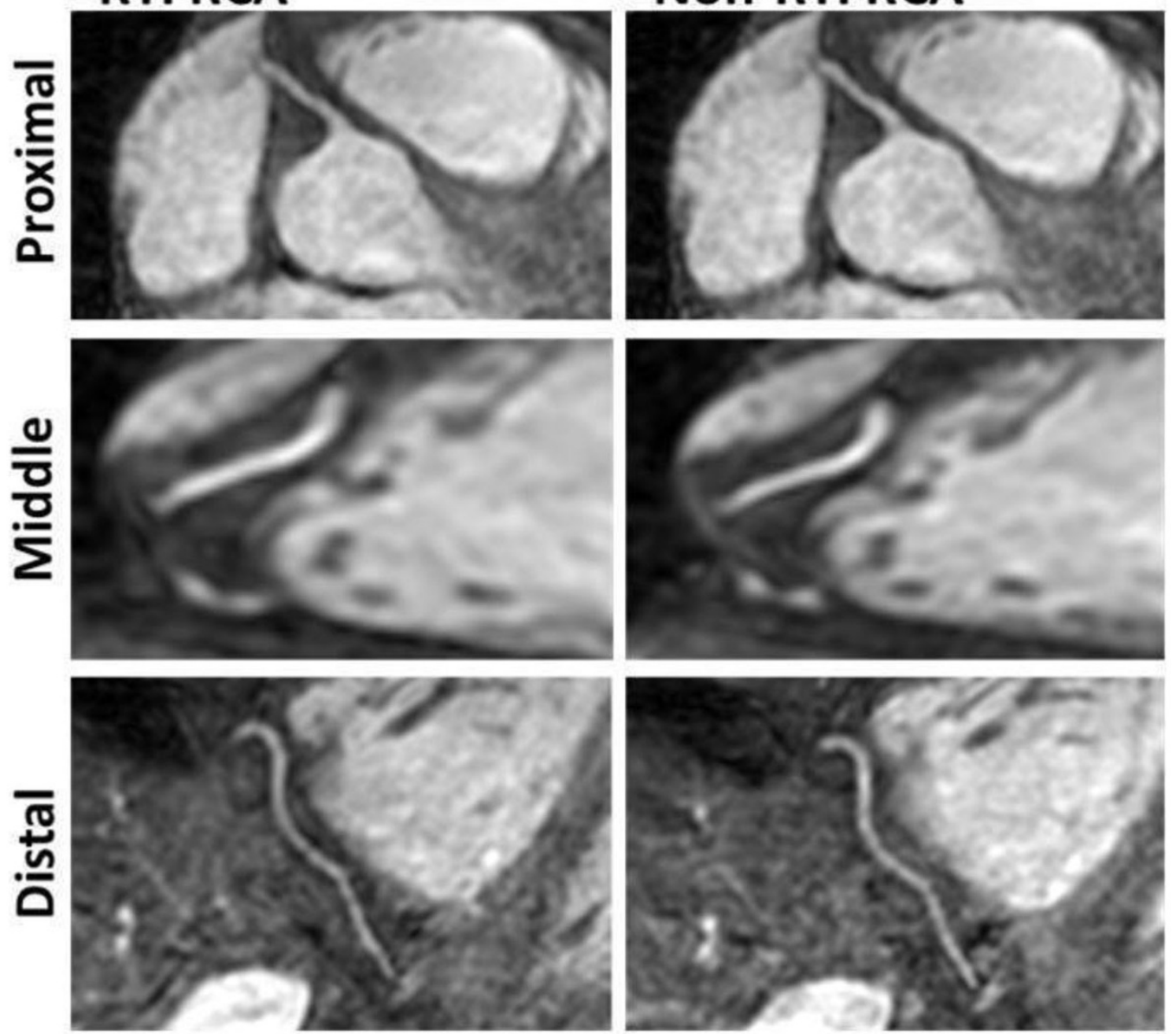

Figure 2 Example of RTI adjusted CCMR compared against a non-RTI approach reference. Top: RTI and non-RTI NAV profiles. RTI: 324 HBs; NavEff $=62 \%$ vs non-RTI: $438 \mathrm{HBs}$ and $46 \%$. Operator interaction involved expanding the navigator gating window size from $5 \mathrm{~mm}$ to $7.5 \mathrm{~mm}$ midway (136th out of $324 \mathrm{HBs}$ ) during the scan. This increased the navigator efficiency from 56\% (first $136 \mathrm{HBs}$ ) to $66 \%$ (Remainig $188 \mathrm{HBs}$ ). Bottom: Acquired RCAs. Sharpness scores were (RTI: 0.52) vs (non-RTI: 0.48). 
weighted navigator gating. 13 healthy volunteers were imaged on a $1.5 \mathrm{~T}$ system (Philips Achieva) using a 5 channel cardiac array. Scan parameters were: TR = $4.4 \mathrm{~ms} ; \mathrm{TE}=1.9 \mathrm{~ms} ; \mathrm{FA}=90 ; 300 \times 300 \times 100-$ $130 \mathrm{~mm}^{3}$ at $1.3 \mathrm{~mm}^{3}$, interpolated to $0.65 \times 0.65 \times$ $1.3 \mathrm{~mm}$ resolution; Sensitive Encoding $(\mathrm{R}=2)$ was used. The default gating window was $5 \mathrm{~mm}$ set by a 20-heartbeat $(\mathrm{HB})$ calibration. Slice tracking was not used. Two volumes were acquired; one employing RTI, and another without using this tool. RTI and non-RTI CCMR acquisitions were randomized. Total number of HBs (calibration HBs not included), navigator efficiency (NavEff), and vessel sharpness in the RCA, LAD, and LCX were measured. Student's t-test was used for statistical analysis.

\section{Results}

The proposed RTI tool allowed successful completion of $3 \mathrm{D}$ coronary acquisition in all 13 subjects (375 \pm $67 \mathrm{HBs}$, NavEff $=56 \pm 9 \%$ ). Figure 2 shows a representative example. The non-RTI scans resulted in the operator restarting the scan in seven subjects $(\mathrm{n}=8$ total restarts; stopped @ $82 \pm 51$ HBs w. NavEff $=26 \pm$ $12 \%$; restart rate $=40 \%[8 / 20$ scans $])$. Of these, non-RTI data was not collected in $\mathrm{n}=1$ due to significant respiratory drifting. The total $\mathrm{HBs}$ for $\mathrm{n}=12$ non-RTI scans were $443 \pm 76(\mathrm{p}<0.001$ vs RTI), with NavEff $=$ $48 \pm 6 \%(\mathrm{p}<0.005$ vs RTI). Sharpness scores (RTI vs non-RTI) were as follows: RCA $(0.48 \pm 0.04$ vs $0.46 \pm$ $0.05 ; \mathrm{p}<0.05)$, LAD $(0.41 \pm 0.06$ vs $0.42 \pm 0.04 ; \mathrm{p}=\mathrm{NS})$, and LCX $(0.40 \pm 0.05$ vs $0.41 \pm 0.04 ; \mathrm{p}=\mathrm{NS})$.

\section{Conclusions}

The feasibility of RTI manipulation between waveform generator and host console during MRI data acquisition was successfully demonstrated without need for additional dedicated research hardware. RTI operator-guided manipulation of the navigator gating window eliminated repeated acquisitions of 3D CCMR sequences in all 13 subjects, while achieving $\sim 70$ fewer HBs, $\sim 8 \%$ NavEff increase, and improved/comparable sharpness compared to conventional non-RTI CCMRs.

\section{Authors' details}

${ }^{1}$ Medicine, Section of Cardiology, The University of Chicago, Chicago, IL, USA. ${ }^{2}$ Philips Healthcare, Best, Netherlands. ${ }^{3}$ Philips Healthcare, Cleveland, $\mathrm{OH}$, USA.

Published: 27 January 2016

\section{Reference}

1. Kawaji, et al: MRM 2014
doi:10.1186/1532-429X-18-S1-P321

Cite this article as: Kawaji et al:: Operator-guided Navigator Gating for Real-Time Interactive Coronary Cardiovascular Magnetic Resonance. Journal of Cardiovascular Magnetic Resonance 2016 18(Suppl 1):P321.
Submit your next manuscript to BioMed Central and take full advantage of:

- Convenient online submission

- Thorough peer review

- No space constraints or color figure charges

- Immediate publication on acceptance

- Inclusion in PubMed, CAS, Scopus and Google Scholar

- Research which is freely available for redistribution

Submit your manuscript at www.biomedcentral.com/submit 